\title{
Generating a Multi-Timeframe Trading Strategy based on Three Exponential Moving Averages and a Stochastic Oscillator
}

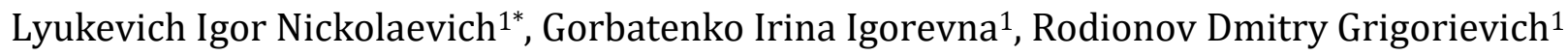 \\ ${ }^{1}$ Graduate School of Industrial Economics, Institute of Industrial Management, Economics and Trade, Peter \\ the Great St. Petersburg Polytechnic University, Politechnicheskaya St., 29, St. Petersburg, 195251, Russia
}

\begin{abstract}
This study combines a fundamental analysis of the rationale for conservative investors' transactions, as well as long-term, low-risk strategies, and a technical analysis of the search for entry points into short-term, high-risk speculation. A hypothesis about the possible adaptation of highrisk foreign-exchange-market strategies to a low-risk stock market, based on a multi-timeframe analysis of the intersection of 3 EMA plus stochastic (a combination of three moving averages and a stochastic oscillator), is proven. The study's modeling is based on walk-forward, blind simulation, cross procedure for realistically testing a hypothesis that can be performed in nine steps (Colby, 2003.) Colby's algorithm Its subject is ordinary shares of Sberbank of Russia, and its analysis shows an absence of uncharacteristic movements in the chosen period of maximum volatility, from 2007 to the present. This analysis was conducted for two timeframes (more than five years for the trend direction and less than three years for the entry point). For the EMA, parameters were set at 20, 50, and 200; for stochastic parameters were set at 14, 3, and 3, 80/20. The "failure swing" reversal pattern and new support and resistance lines were detected. The study's main conclusions are that the simultaneous use of three EMAs makes determining a corridor or a trend fairly reliable, as well as setting stop-losses. Moreover, the use of an oscillator is found not to always be reasonable; its main task is to confirm a signal. A stochastic oscillator with an explicit trend should not be analyzed for the whole period under consideration-only the last values should be considered. Moving averages and oscillators give fewer false signals on medium-term timeframes than on short-term timeframes. Due to a change in trend direction, identifying new (defined and correct) support and resistance lines is found to be necessary.
\end{abstract}

Keywords: Algorithmic trading; Financial market; Moving averages; Stochastic; Technical indicators; Trading strategies

\section{Introduction}

Digital technologies' role in the modern circular economy is becoming more and more important (Berawi, 2020). Studies have shown a link between basic human values and risk perceptions, between risk perceptions and risk behavior, and between risk behavior and accidents (Sutalaksana, 2019). Therefore, an important task is to minimize the emotional component of production processes and, in particular, to algorithmize the search for the right trading strategies.

The development of forecast price models is one of the most active application

*Corresponding author's email: lyukevich_in@spbstu.ru, Tel.: +7-905-2038110

doi: 10.14716/ijtech.v11i6.4445 
Areas (Plotnikova, 2018) .Basic models have similar objectives, based on minimizing overall costs and increasing the efficiency of available resources (Balashova and Gromova, 2017).

The aim of the current work is to create a new trading strategy for the stock market, based on well-known technical indicators. The foreign exchange market is volatile; only professional traders can accept high risks, and their strategies aim to clearly define entry and exit points. To generate this study's strategy, the authors took a simple forex strategy as a basis and adapted it to the stock market for conservative investors. Our working hypothesis is that the trading strategy for a low-risk stock market can be improved by adapting high-risk forex strategies.

Multi-frame analysis is an assessment of a given situation from different timeframesa skill that many market participants lack. (Singh, 2000) And this lack is significantly problematic because many investors do not consider emerging graphical patterns in global trends or the impact these patterns can have on market participants' trading decisions in other timeframes. Thus, our explanatory hypothesis is that adapting strategies is possible on the basis of multi-timeframe analysis, in which a longer timeframe determines a trend's direction and a shorter timeframe determines an optimal entry point.

\section{Literature Review}

The theory of forecasting price trends is based on publications by Dow, the author of the oldest market index. Most of Dow's theories were formulated by Hamilton (1922) and practically developed by Rhea (1932). Wave patterns were suggested and mathematically justified by Elliott (1938). This theory gained power in the 1980s, thanks to Prechter and Frost (1991).

Gann (1941) offered a fundamentally different approach to financial market forecasting, based on geometry, numerology, and ancient mathematics, astronomy, and astrology (Mitaeva). Two more works-by Clement (1908) and McWhirter (1938)-are considered important in this area, too, but the authors are probably aliases of Gann himself. No unified version of Gann's trading system exists, and modern researchers are trying to systematize several of his works (Mikula, 1995).

Technical analysis, as a science of studying market dynamics via charts to predict price movements' future direction, was initiated by Murphy (1986). However, perhaps the first textbook on technical analysis based on price candlesticks is a Japanese book of 1755 by Honma, The Three Monkey Record of Money (Gusev, 2015), but Japanese candlesticks have become very popular, thanks to the work of Nison (1994). Yet, the "tic-tac-toe" method of keeping price charts and its corresponding forecasting principles were established by Villiers (1933).

Our model is based on Williams' trading paradigm of waiting for the beginning of a strong stable trend and then, after a suitable trend is detected, searching for entry and exit points (Williams, 1979). Our basic strategy-3 EMA plus stochastic (a combination of three moving averages and a stochastic oscillator) is not very popular, but it is often used on the forex market for short timeframes (Forex Strategies, 2014).

Moving averages are one of the oldest and most widely used tools for technical analysis (Achelis, 1995). Stochastic optimization is a scientific methodology for maintaining a system's operation in security and safety. This calculation provides an important indicator with which to analyze risks in a system under operational conditions (Bouayoune, 2017). This trading analysis methodology was first described in 1957 (Schade, 2005). Lane contributed significantly to the stochastic oscillator's recognition and popularity as a technical indicator (Lane, 1985). 
Due to the "mathematization" of finance, more and more complex models are developed to better approximate price movements, using historical data and including economic indicators as factors (Skhvediani and Kudryavtseva, 2018). A direction of robotizing trading, based on the blockchain, seeks to exclude the "human being" and human factor from the decision-making system (Babkin et al., 2018). Different methods of formalizing information flows have been proposed as optimal strategies (Kalmykova et al., 2017). Innovative financial online platforms have also been developed (Bataev, 2018). A separate area is analyzing the cryptocurrency market (Arutunyan et al., 2018).

A growing literature is examining and generating trading strategies. In the field of research, authors creating strategies use rules from the popular technical indicators slope, exponential moving average (MA), moving average convergence divergence, relative strength index, stochastic oscillator, and directional index (Faijareon and Sornil, 2019). Huang and Huang (2020) proposed a quasi-intraday version of the standard MA strategy, QUIMA. Naik and Mohan (2019) considered 33 different combinations of technical indicators to predict stock prices, finding a way to select the relevant technical indicators accurately and creating a prediction model for stocks.

\section{Methods}

Future market behavior will largely repeat the patterns of the past (Kuporov et al., 2018). Calculating a time series' future values, based on these values' functional dependence on the already-specified time values, is necessary.

Our modeling is based on Colby's algorithm: (1) hypothesis development; (2) data collection; (3) data verification for uncharacteristic movements; (4) data segmentation into time intervals; (5) indicator parameter search; (6) indicator testing; (7) expansion of a reliable segment; (8) multiple repetitions of stages 5,6 , and 7 to completely remove invalid data; and (9) evaluating results (Colby, 2003). We selected the TradingView platform for this study (TradingView).

Indicators for creating our model. The key financial-market factors are the price of an asset and the volume of its trading or open interest. Let us assume as a basis that the proposed strategy should consider both of these factors.

The first factor is trend indicators that measure a trend's direction and strength by comparing prices with a set baseline. They will help determine support and resistance levels, find patterns, and predict trend reversals. We chose exponential moving averages (EMAs). Current price data affect a moving average exponentially more than SMAs, where the same weight is assigned to all values. For more details about exponential smoothing in economic forecasting, see the work of Lyukevich (2018)

The second factor is volume indicators that estimate oversold or overbought assets, oscillators. The signal, in this case, is the change in a price growth rate under an existing trend. The stochastic oscillator is the most important oscillator (Lebeau and Lucas, 1992).

\section{Results}

To succeed in the financial market, each participant-based on their risk appetite and available time-determines a trading strategy appropriate for them. Personal identity is taken into account; for one investor, a strategy can work very well, but for another, the same strategy can be absolutely unacceptable (Lebedev et al., 2018).

Choice of strategy. We suggest a multi-timeframe strategy of crossing three EMAs plus a stochastic oscillator. The simultaneous crossing of three EMAs can be used as a separate strategy that allows for a filtering-out of considerable market noise, but-as 
with standard, slightly lagging trend indicators - it comes with the cost of having to slightly miss the beginning of a trend. For this reason, the strategy of crossing three exponentially moving averages in combination with an anticipatory instrument, which is a stochastic oscillator in this case, provides good results (Figure 1).

\begin{tabular}{|c|c|c|}
\hline Trends & Volumes & Time Lags \\
\hline$\downarrow$ & $\downarrow$ & $\downarrow$ \\
\hline Moving Averages & Oscillators & Timeframes \\
\hline$\downarrow$ & $\downarrow$ & $\downarrow$ \\
\hline EMA $(20,50,200)$ & Stochastic $(14,3,3 ; 80 / 20)$ & $\begin{array}{l}\text { No } \\
\text { Higher time-frame: } 5 \text { years } \\
\text { Lower time-frame: } 3 \text { years }\end{array}$ \\
\hline$\downarrow$ & $\downarrow$ & $\downarrow$ \\
\hline
\end{tabular}

Figure 1 Multi-timeframe strategy scheme based on three exponential moving averages and a stochastic oscillator

We open long positions when two EMAs with shorter periods cross the EMA with the largest period from bottom to top. We exit the position when a slow stochastic line crosses the set overbought level from top to bottom. Additional signals for exiting are a candlestick closing below or above the EMA with the largest period.

Data collection. To test a trading strategy, step-by-step modeling of the strategy is necessary, based on historical data. We chose the ordinary shares of Sberbank of Russia as the subject of our strategy modeling. The bank's share price tends to grow globally. This tendency leads to increased demand for these securities (Figure 2). The price changed by 1,000 times, while the number of shares among shareholders increased by 1,000 times, correspondingly. This split took place just before the world financial crisis in 2008 (Figure 3).

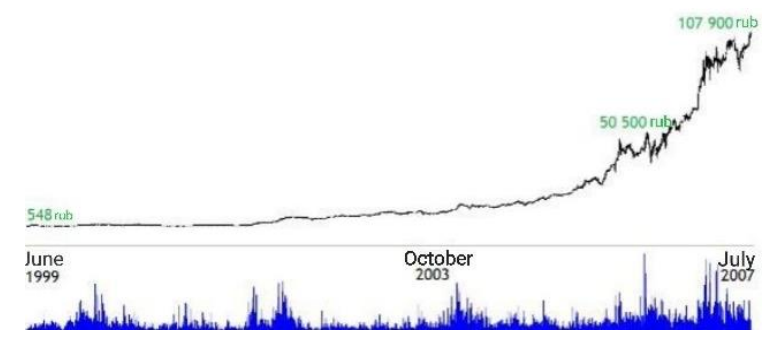

Figure 2 Share price before the 2007 split

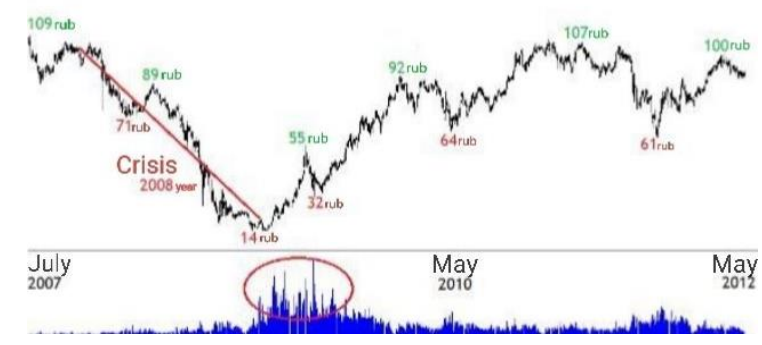

Figure 3 Share price changes after the 2007 split

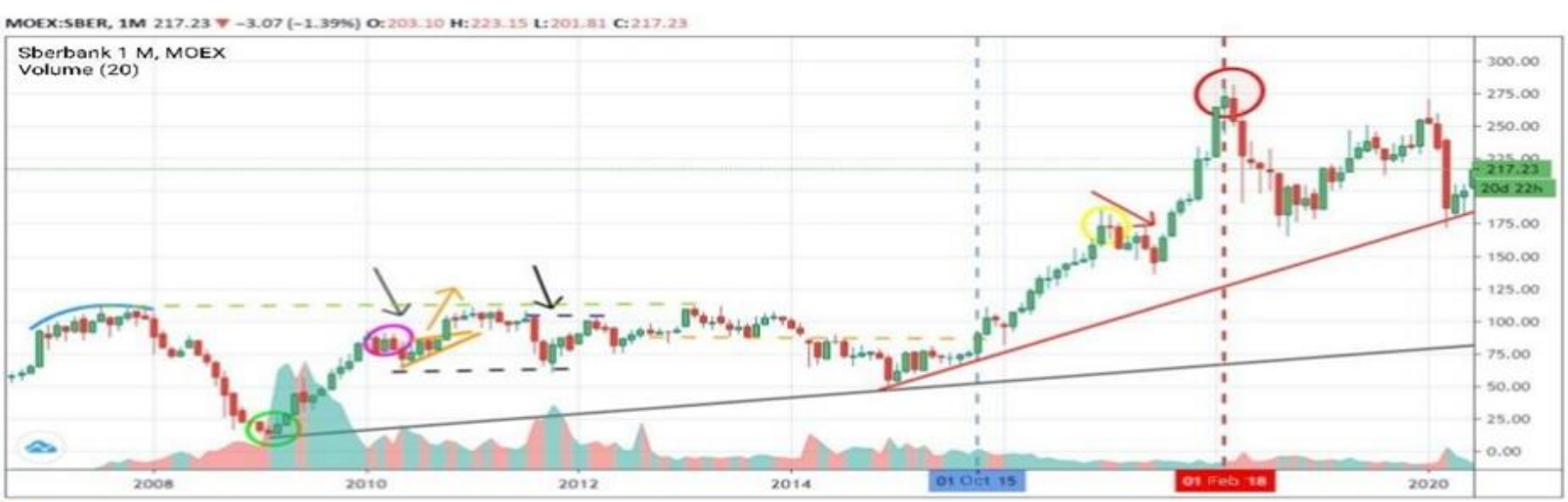

Figure 4 Dynamics of ordinary Sberbank share prices for the 2007-20 period 
A data check showed no uncharacteristic movements. Conducting an analysis over the long term in the early periods, when market price volatility was minimal, is unnecessary, so the time period from 2007 to the present was chosen (Figure 4).

The 2008-09 period was characterized by a significant price drop, from 109 to 14 rubles, which-from a fundamental point of view-was due to the beginning of the global financial crisis. A downward price reversal foreshadowed the formation of the "reverse saucer" pattern (the blue line in the left part of the chart in Figure 4).

March 2009 to January 2010 marked a phase of active price growth on the market, which was accompanied by a very large volume.

The phase of active price growth stopped, and market consolidation began, forming a sideways trend from February to April 2010 (the pink circle on the chart in Figure 4). During this time, stocks do not break through their local resistance levels, which causes a drop in purchase demand.

In the second half of 2010, the sideways trend was replaced by an upward movement. Updated local support and resistance lines formed a "rising triangle" pattern (marked by orange lines on the chart in Figure 4), which gave market participants a signal that the resistance line of the triangle had broken and opening long positions was possible. By the beginning of 2011, the bank's shares reached their historical maximum of 2007.

Furthermore, in the first half of 2011, prices remained in a narrow range, and the market had a long sideways trend. As a result, prices reached a level similar to the decline in 2010 (marked by a black dotted line on the chart in Figure 4). This change confirms that "history repeats itself."

The beginning of 2013 was characterized by a repetition of the historical peaks of 2007 and 2011 (marked by a green dotted line on the chart in Figure 4). Later, in 2013, the bullish trend weakened, and the share price could not break through its resistance level. At the beginning of 2014, the price sharply declined, after which the support level was the resistance level (marked by an orange dotted line on the chart in Figure 4).

The recovery of the upward global trend began in October 2015. A phase of active price growth continued until the end of 2016. After reaching a peak of 185 rubles (marked by a yellow circle on the chart in Figure 4), the price was corrected by a local downtrend (marked with a red arrow). Then came a second wave of very active growth, which ended in February 2018 with a renewal of the historical maximum whose price level was 285 rubles per share (marked by a red circle and a red vertical line on the chart in Figure 4). This level is still relevant today.

Data segmentation into time intervals. Trend is a fractal structure, the global consists of local. So, limiting to just one timeframe, a lot of information is missed. A longer timeframe is needed to determine a trend's direction the most accurately, while an analysis of a shorter time interval is used to find an optimal entry point. Let us agree that the model should contain two subsystems: the first assesses "knowledge," and the second assesses "commercialization" (Rudskaya and Rodionov, 2018). Let us set two timeframes: long-term (five years, the period from 2015 to 2020) and medium-term (three years, the period from 2017 to 2020).

Indicator parameter search. The chosen forecasting strategy implies the use of exponential moving averages and a stochastic oscillator. For the EMA, we set the parameters 20 (short period; the orange line in Figure 5), 50 (average period; the blue line in Figure 5), and 200 (long-term period; the green line in Figure 5). For the stochastic oscillator, the period can be set to any value. We chose recommendations for smooth 
charts (period: 14; moving average: 3 ; and smoothing: 3 [14, 3, 3]). Overbought and oversold levels are in the ratio of $80 / 20$.

Indicator testing. The share price tended to grow in 2015-2020 period (Figure 4). The historical maximum was reached in February 2018, and it is the absolute resistance level at the moment (marked with an orange dotted line in Figure 5). From 2017 to 2020, the "failure swing" reversal pattern (marked by a rectangular area), with peaks going down (lines 1 and 2 in Figure 5), was clearly lined up. The fact that the trend's strength had weakened is also evidenced by an increase in the volume of transactions in the fall, after reaching highs (marked by the orange circles in Figure 5). This fact should be considered in order to analyze a shorter time interval.

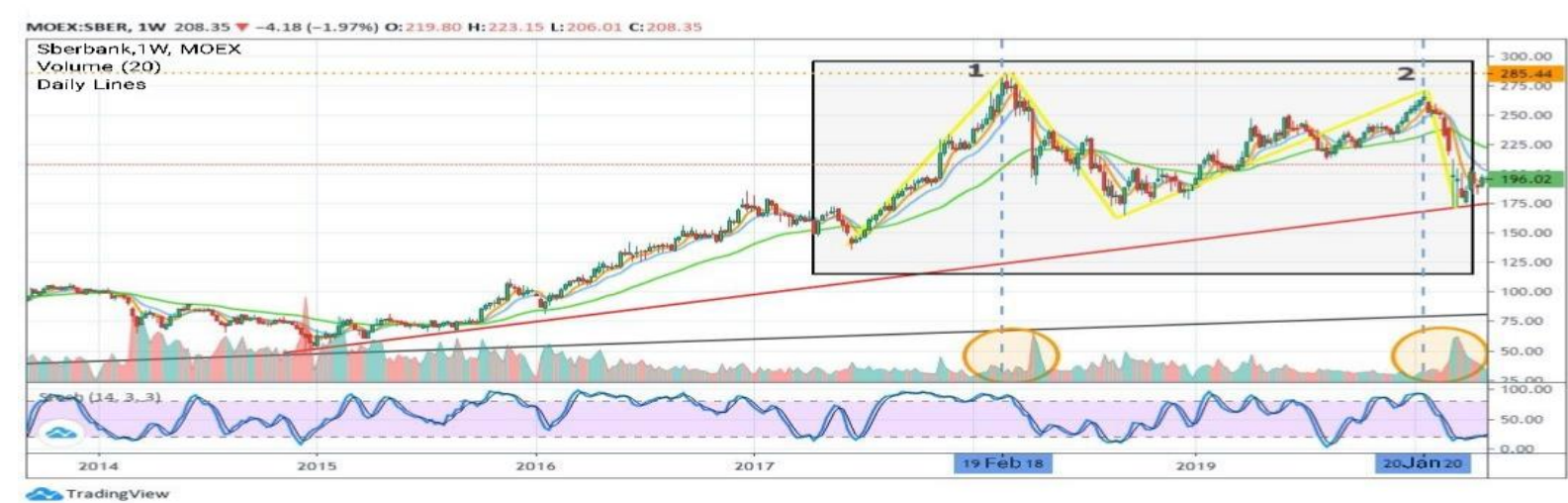

Figure 5 Graphical pattern identification

Several factors became growth drivers for the share price: favorable market conditions for the industry, a strong position for the issuer within the industry, a high oil price that ensured an inflow of cash into the banking sector, the reliability of capital investment due to the issuer's high rating, and the new dividend policy (a strategy for 2017-20), which attracted long-term investors, growing profits.

Expansion of the reliable segment. Today, the shares tend to descend (Figure 5). The price has broken through its support line (Line 1 in Figure 5), which had existed since 2015 and was very significant. In addition, as a result of breaking this line, a price gap formed from 228 to 197 rubles (March 2020), which signaled new lows and was also confirmed by an increase in the volume of transactions during this period-that is, most market participants supported the new local downtrend. At the moment, the price has formed a new support line (Line 2 in Figure 5) and a local resistance line (Line 3 in Figure $5)$.

Exponential moving averages with a short period (EMA 20, the orange line; EMA 50, the blue line; Figure 5) crossed the moving average with the largest period (EMA 100, the green line; Figure 5) from top to bottom, which was a "sell" signal. This crossing happened before the local gap formed and prices fell. Here, the signal included all three EMAs' being under the price chart. The trend indicator confirms that the trend is weak.

The stochastic oscillator should not be analyzed for the entire period under consideration if a clear trend is evident. In this context, considering only the last values is necessary. In April 2020, the indicator was in the overbought zone (the red vertical line in Figure 6) at 92, after which a fall in prices was observed. At the moment of this analysis (May 2020), the stochastic oscillator is neutral at 52.

Multiple repetition. On the basis of our analysis, we can predict two options for the outcome of events for this share. 


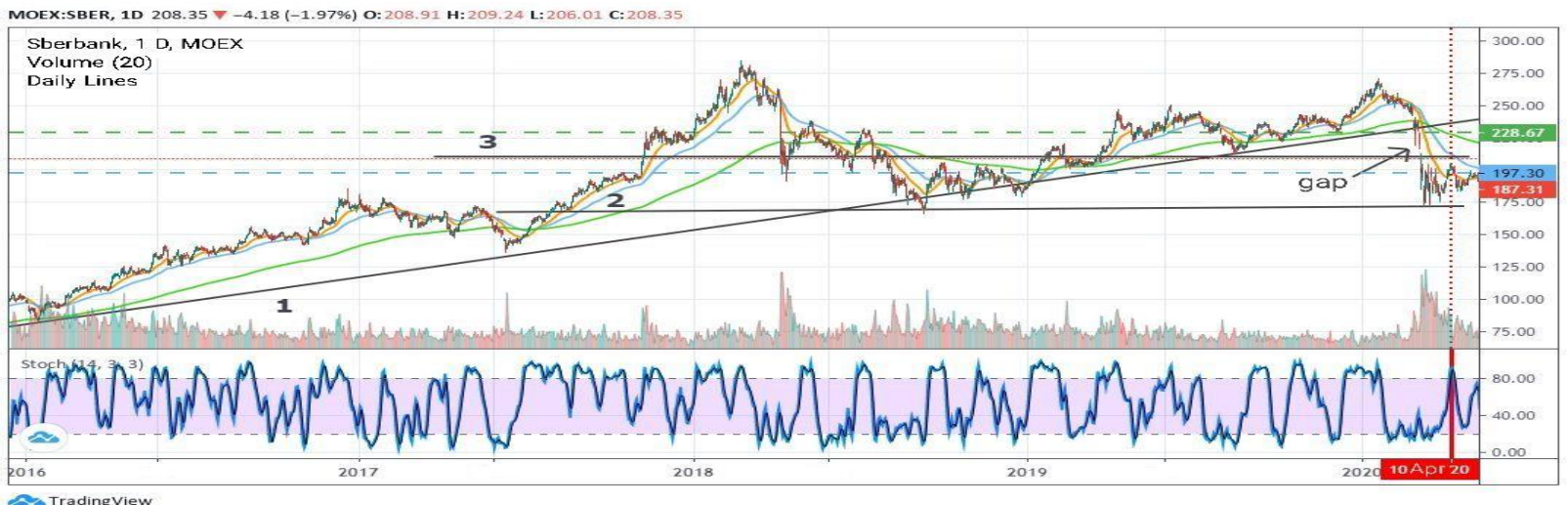

Figure 6 Current situation on the market for Sberbank shares

First, given the high probability of a global trend reversal, the price will continue to break through its support levels and strive for its most significant level, which formed in 2009. Thus, the worst projected asset price will be at the 80-90 ruble level. The confirmation that this option is more likely is the stochastic oscillator's not having reached the overbought zone's boundary. In this case, the indicator shows relative equality between supply and demand for the shares under analysis. In addition, all three EMAs are located in an inherent position for a downtrend (the long EMA [200] at the top; the short EMA [20] at the bottom) and have a strong slope.

Second, due to the weakness of the global upward trend, the share price will go into a trading range limited by lines 2 and 3 for a period. Breaking through this range of 170210 rubles is possible if fundamental macroeconomic news has a positive connotation for investors. From a technical point of view, the price may break this range from bottom to top in order to "close the gap" - that is, attempting to trade the range with the upper price level of 228 rubles is locally probable. After fixing the price at this level, we expect the share price will be in the range of 230-240 rubles. In the longer term, we expect market stagnation at best, and in the short-to-medium term, local highs are very likelywhich both the technical and fundamental points of view explain. As a negative factor, any new outbreak is likely to occur as the economy opens up, which would again lead to restrictions and market decline.

Evaluating results. Having analyzed the application of the multi-timeframe strategy of crossing 3 EMA plus stochastic for highly liquid "blue chips" in the financial market of a country with country risk classifications of " 3 ," provided that the shares are more in trend areas, we find the following:

1. The use of an oscillator is not always reasonable; its main task is to confirm a signal.

2. The use of three EMAs allows to determine of whether they are trading in a corridor or a trend, and it also provides an opportunity to set stop-losses without additional calculations (under the longest EMA).

3. Having lined up in a trend line, MAs form a kind of support (resistance) line, marking the end of a correction clearly enough. Thus, a market participant during correction moments can predict which point a price will reach during a correction. A long MA breakthrough provides a strong trend reversal signal.

4. Moving averages and oscillators provide fewer false signals on medium-term charts than on short-term charts.

5. Due to the change in a trend's direction, identifying new (determined and correct) support and resistance lines is necessary. 


\section{Conclusions}

The stochastic oscillator is a leading indicator, and EMAs are lagging indicators. The search for the optimal parameters for these indicators will allow a balance of the market signals, which will increase the trading strategy's effectiveness. We have found the parameters that allow for an adaptation of high-risk forex market trading strategies to the stock market. First, these parameters include two timeframes: the higher timeframe is five years - for the trend direction-while the lower timeframe is three years-for the entry point. Second, a combination of three EMAs (20, 50, and 200 daily) plus stochastic provides a main (fast) line averaging over 14 and smoothing over three periods, as well as a signal (a slow-moving average after a fast-moving average) line with a period averaging 3 , with $80 \%$ overbought and $20 \%$ oversold shares. Third, combining EMAs as a trend indicator with a stochastic oscillator as a market speed indicator allows for the identification of patterns and the determination of the optimal points to open and close positions.

The specific quantitative results are two probable forecasts that indicate specific price levels at which to enter the market: (1) 80-90 rubles per share, and (2) 230-240 rubles per share. At the moment (November 2020), the share price is 238 rubles per share, which confirms our strategy's reliability and potential for practical application.

Discussion. A strategy's effectiveness can be assessed by its ratio of true and false signals, compared with other strategies for similar timeframes. The fewer false signals, the more effective the strategy. The continuation of the study in testing the strategy with other indicator parameters to find the best combination.

The resulting forecast model is designed for a wide range of people; it is comprehensible and easy to use. This approach is how a trading algorithm can be generated and programmed for automated trading systems (per MetaTrader, InteractiveBrokers, and others) and developing a robo-advisor.

\section{Acknowledgements}

This research was supported by the Academic Excellence Project 5-100, proposed by Peter the Great St. Petersburg Polytechnic University.

\section{References}

Achelis, S.B., 1995. Technical Analysis from A to Z: Every Trading Tool...from the Absolute Breadth Index to the Zig Zag. New York: McGraw-Hill Book Company

Arutunyan, M., Skhvediani, A., Kudryavtseva, T., Novikov, S., 2018. ARIMA Model for Describing Dynamics of Bitcoin Cryptocurrency. In: Proceedings of the $32^{\text {nd }}$ International Business Information Management Association Conference 2018

Babkin, A.V., Burkaltseva, D.D., Betskov, A.V., Kilyaskhanov, H.S., Tyulin, A.S., Kurianova, I.V., 2018. Automation Digitalization Blockchain: Trends and Implementation Problems. International Journal of Engineering and Technology, Volume 7(3), pp. 254-260

Balashova, E.S., Gromova, E.A., 2017. Russian Experience of Integrating Modern Management Models. Espacios, Volume 38(53), pp. 31-39

Bataev, A.V., 2018. Innovative Forms of Interaction between Financial Institutions and Clients: Automated Banking Offices. In: Proceedings of the $3^{\text {rd }}$ International Conference Ergo-2018: Human Factors in Complex Technical Systems and Environments, Ergo, pp. 9-13

Berawi, M.A., 2020. Managing Nature 5.0: The Role of Digital Technologies in the Circular Economy. International Journal of Technology, Volume 11(4), pp. 652-655 
Bouayoune, K.S., Boudi, E.M., Bachir, A., 2017. A Stochastic Method based on the Markov Model of Unit Jump for Analyzing Crack Jump in a Material. International Journal of Technology, Volume 8(4), pp. 622-633

Clement, L., 1908. The Ancient Science of Numbers. New York: Roger Brothers

Colby, R.W., 2003. The Encyclopedia of Technical Market Indicators. $2^{\text {nd }}$ ed. McGraw-Hill Publishing

Elliott, R.N., 1938. The Wave Principle. Alanpuri Trading, Los Angeles, CA, 2013 (originally published by R.N. Elliot, New York, NY)

Faijareon, C., Sornil, O., 2019. Evolving and Combining Technical Indicators to Generate Trading Strategies. Journal of Physics, Conference Series 1195, pp. 16-32

Forex Strategies, 2014. Stochastic Scalping with Three Moving Averages. Available Online at https://www.forexstrategiesresources.com/scalping-forex-strategies-ii/281stochastic-scalping-with-three-moving-averages

Gann, W.D., 1941. How to Make Profits Trading in Commodities: AA Study of the Commodity Market. Lambert-Gann, Pomeroy, WA

Gusev, V.P., 2015. Japanese Candles. Application Features. [s. 1.] Moscow

Hamilton, W.P., 1922. The Stock Market Barometer: A Study of Its Forecast Value Based on Charles H. Dow's Theory of the Price Movement: With an Analysis of the Market and Its History Since 1897. New York: Harper \& Bros

Huang, J.-Z., Huang, Z., 2020. Testing Moving Average Trading Strategies on ETFs. Journal of Empirical Finance, Volume 57, pp. 16-32

Kalmykova, S.V., Pustylnik, P.N., Razinkina, E.M., 2017. Role Scientometric Researches' Results in Management of Forming the Educational Trajectories in the Electronic Educational Environment. Advances in Intelligent Systems and Computing, Issue 545, pp. 427-432

Kuporov, J.J., Kudryavtseva, T.J., Gorovoy, A.A., 2018. Algorithm for Formation of the Investment Project Portfolio of a Public Utility Company. Proceedings of the $31^{\text {st }}$ International Business Information Management Association Conference, 2018

Lane, G.C., 1985. Lane's Stochastics: The Ultimate Oscillator. CMT Association Journal (Journal of Technical Analysis), Issue 21, pp. 37-42

Lebeau, C, Lucas, D.W., 1992. Technical Traders Guide to Computer Analysis of the Futures Markets. McGraw-Hill Education

Lebedev, O.T., Mokeeva, T.V., Rodionov, D.G., 2018. Matrix Structures of Science and Technology Innovations Development and Implementation Trajectory. In: Proceedings of the $31^{\text {st }}$ International Business Information Management Association Conference

Lyukevich, I., Agranov, A., Kulagina, N., 2018. Issues of Exponential Smoothing in Economical Forecasting. In: Proceedings of the $32^{\text {nd }}$ International Business Information Management Association Conference, 2018

McWhirter, L., 1938. Astrology and Stock Market Forecasting. New York: ASI Publishers Inc (Second ed., 1977)

Mikula, P., 1995. Gann's Scientific Methods Unveiled. Volume 1 and Volume 2. P. Mikula Pub. and Trading, Austin, USA.

Mitaeva, 0., (n/d) Technical Analysis: The Mysterious Methods of William Delbert Gunn. Available online at Educational Site for Investors and Traders. https://itrading.ru/poleznoe/izvestnye-trejdery/vilyam-delbert-gann

Murphy, J.J., 1986. Technical Analysis of the Futures Markets: A Comprehensive Guide to Trading Methods and Applications. New York Institute of Finance

Naik, N., Mohan, B.R., 2019. Optimal Feature Selection of Technical Indicator and Stock Prediction using Machine Learning Technique. In: Emerging Technologies in Computer 
Engineering: Microservices in Big Data Analytics. ICETCE 2019., Volume 985, pp. 261268, Singapore: Springer

Nison, S., 1994. Beyond Candlesticks: New Japanese Charting Techniques Revealed. John Wiley \& Sons

Plotnikova, E.V., 2018. Investigating the Influence of Gender and Age on the Choice of Housing. In: Proceedings of the $31^{\text {st }}$ International Business Information Management Association Conference, 2018

Prechter, R.R. Jr., Frost, A.J., 1991. Elliott Wave Principle: Key to Stock Market. New York: McGraw Hill Publishing Co.

Rhea, R., 1932. The Dow Theory: An Explanation of Its Development and an Attempt to Define Its Usefulness as an Aid in Speculation. New York: Barron's

Rudskaya, I.A., Rodionov, D.G., 2018. Comprehensive Evaluation of Russian Regional Innovation System Performance using a Two-Stage Econometric Model. Espacios, Volume 39(4), pp. 40-52

Russell, R., 1961. The Dow Theory Today. New York: Richard Russell Associates, New York

Schade, G., 2005. The Origins of the Stochastic Oscillator. The Chartered Market Technician (CMT) Association. Available Online at https://cmtassociation.org/kb/origins-of-thestochastic-oscillator-article

Singh, S.P., 2000. Modelling in Time-Series Forecasting. Cybernetics and Systems-An International Journal, Volume 31(1), pp. 49-65

Skhvediani, A.E., Kudryavtseva, T.Y., 2018. The Socioeconomic Development of Russia: Some Historical Aspects. European Research Studies Journal, Volume 21(4), pp. 195207

Snezhko, Y.S., 2015. The Use of Technical Analysis Indicators in the Russian Stock Market. Russian Journal of Entrepreneurship, Volume 16(16), pp. 2681-2696

Sutalaksana, I.Z., Zakiyah, S.Z.Z., Widyanti, A., 2019. Linking Basic Human Values, Risk Perception, Risk Behavior and Accident Rates: The Road to Occupational Safety. International Journal of Technology, Volume 10(5), pp. 918-929

TradingView. (n/d) Free Stock Charts, Stock Quotes and Trade Ideas. Available Online at https://www.tradingview.com

Villiers, V.d., 1933. The Point and Figure Method of Anticipating Stock Prices: Complete Theory \& Practice, Windsor Books, Brightwaters, New York: reprinted in 1975

Williams, L.R., 1979. How I Made One Million Dollars Last Year Trading Commodities. Windsor Books 